\title{
An Optimal Constraint Programming Approach to the Open-Shop Problem
}

\author{
Arnaud Malapert \\ École des Mines de Nantes, LINA UMR CNRS 6241, Nantes, France \\ École Polytechnique de Montréal, CIRRELT, Montréal, Québec, Canada \\ arnaud.malapert@mines-nantes.fr \\ Hadrien Cambazard \\ Cork Constraint Computation Centre, Department of Computer Science, University College Cork, Ireland \\ Christelle Guéret \\ École des Mines de Nantes, IRCCyN UMR CNRS 6597, Nantes, France \\ Narendra Jussien \\ École des Mines de Nantes, LINA UMR CNRS 6241, Nantes, France \\ André Langevin, Louis-Martin Rousseau \\ École Polytechnique de Montréal, CIRRELT, Montréal, Québec, Canada
}

This paper presents an optimal constraint programming approach for the Open-Shop scheduling problem, which integrates recent constraint propagation and branching techniques with new upper bound heuristics. Randomized restart policies combined with nogood recording allow to search diversification and learning from restarts. This approach is compared with the best-known metaheuristics and exact algorithms, and shows better results on a wide range of benchmark instances.

Key words: Production-Scheduling, Open shop; Computers-computer science: Artificial Intelligence; Constraint Programming; Randomization and Restart

\section{Introduction}

Open-Shop problems are at the core of many scheduling problems involving unary resources such as Job-Shop or Flow-Shop problems, which have received an important amount of attention because of their wide range of applications. Among the many techniques proposed in the literature, Constraint Programming (CP) is among the most successful.

In the Open-Shop problem (OSP), a set $J$ of $n$ jobs, consisting each of $m$ tasks (or operations), must be processed on a set $M$ of $m$ machines. The processing times are given by a matrix $P: m \times n$, in which $p_{i j} \geq 0$ is the processing time of task $T_{i j} \in T$ of job $J_{j}$, to be done on machine $M_{i}$. The tasks of a job can be processed in any order, but only one at a time. Similarly, a machine can process only one task at a time. We consider the construction of non-preemptive schedules of minimal makespan $C_{\max }$, which is NP-Hard for $m \geq 3$ (see Gonzalez and Sahni, 1976). Figure 1 shows an optimal solution of the problem where each line/shade represents a machine/job.

A classical lower bound $C_{\max }^{L B}$ for this problem is equal to the maximum workload over every machine and every job given by: $\max \left(\max _{1 \leq i \leq m}\left(\sum_{j=1}^{n} p_{i j}\right), \max _{1 \leq j \leq n}\left(\sum_{i=1}^{m} p_{i j}\right)\right)$. Three sets of benchmark instances are available in the literature (Taillard, 1993; Brucker et al., 1997; Guéret and Prins, 1999). 


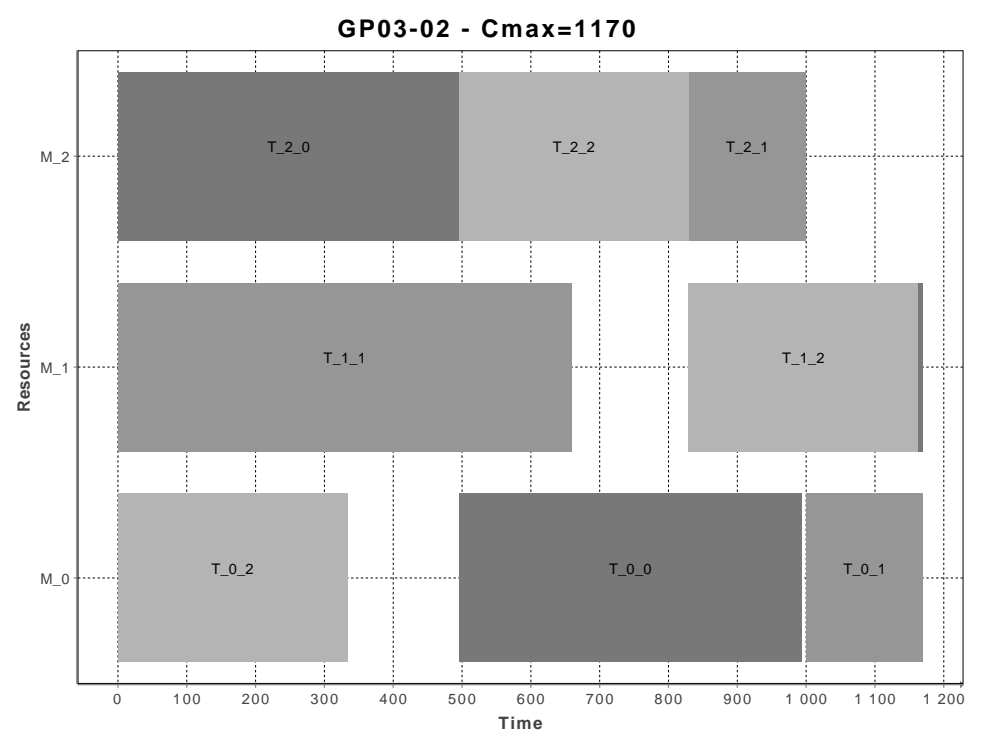

Figure 1: An optimal solution of the instance GP03-02 with makespan equal to 1170.

The first exact method (Brucker et al., 1996) proposed for the Open-Shop problem is based on the resolution of a one-machine problem with positive and negative time lags. The second one (Brucker et al., 1997) consists of fixing precedences on the critical path of heuristic solutions computed at each node. Although that last method is efficient, some problems of Taillard benchmark from size $7 \times 7$ remained unsolved.

Guéret et al. (2000) proposed an intelligent backtracking technique applied to the Brucker branching scheme. When a contradiction is raised during search, instead of systematically backtracking to the previous decision (chronological backtracking), the algorithm analyzes the reasons for the contradiction to avoid questioning decisions that are not related to the failure, and backtracks to a more relevant choice point. This approach significantly reduces the number of backtracks, but can consume twice the CPU time in each node. They further applied additional search tree reduction based on forbidden intervals, i.e. time intervals in which no operation can start or end in an optimal solution (Guéret and Prins, 1998).

Dorndorf et al. (2001) improved the Brucker algorithm by using consistency techniques. Instead of analyzing and improving the search strategies, they focused on constraint propagation techniques for reducing the search space. Furthermore, they studied top-down and bottom-up optimization procedures depending on the average workload of a problem instance. The top-down procedure starts with a upper bound $u b$ and tries to improve it. The bottomup procedure starts with a lower bound $l b$ as target upper bound which is incremented by one unit until the problem becomes feasible. Their algorithms were the first to solve many problem instances to optimality in a short amount of time. However, some problems of Taillard benchmark from size $15 \times 15$ remained unsolved, as well as some of Brucker instances from size $7 \times 7$.

More recently, Laborie (2005) proposed a bottom-up search for cumulative scheduling based on the detection of Minimal Critical Sets (MCS) and advanced propagation with self-adapted shaving. A MCS is a minimal set of resource requirements on the same resource that would over-consume the resource if executed simultaneously. A heuristic selects critical sets by 
estimating the related reduction of the search space. The approach closed the 34 remaining open instances of Guéret-Prins benchmark and 3 of the 6 open instances of Brucker benchmark.

Most bottom-up variants differ by the way infeasibilities are resolved. Dichotomous variants resolve infeasibilities by repeatedly dividing the makespan interval $[l b, u b[$ in half until it becomes empty. If the subproblem $\left[l b,\left[\frac{l b+u b}{2}\right][\right.$ is feasible, then the current makespan provides a new upper bound. Otherwise, the value $\left\lceil\frac{l b+u b}{2}\right\rceil$ is a new legal lower bound. As a consequence, these variants begin with a legal lower bound and an initial feasible solution. For instance, the first branch of the first iteration can provide an initial solution if no initial constraint on the makespan is stated. In practice, it is often important to guarantee the length of the initial interval since the number of subproblems depends on it. Therefore, another approach consists in increasing the target lower bound by $2^{k}$ from the previously stored lower bound at the $k$-th iteration until finding a solution which provides the initial interval $\left[2^{k}, u b\left[\subseteq\left[2^{k}, 2^{k+1}[\right.\right.\right.$. Dichotomous variants reduce the number of iterations from $C_{\max }-C_{\text {max }}^{L B}+1$ to $O\left(\log _{2}\left(C_{\max }-C_{\max }^{L B}+1\right)\right)$.

Later, Tamura et al. (2006) applied a method to Open-Shop problems that encodes Constraint Satisfaction/Optimization problems with integer linear constraints into a Boolean Satisfiability Testing problem (SAT). A comparison $x \leq a$ is encoded by a different boolean variable for each integer variable $x$ and each integer value $a$. Then, a simple constraint model with deadline constraints and binary disjunctive constraints between two activities belonging to the same job or machine is encoded as a SAT. They proved optimal results for all instances including the last three open instances of Brucker benchmark.

Many metaheuristics algorithms have been developed in the last decade to solve the Open-Shop problem. The most recent and successful metaheuristics are: Genetic Algorithm (Prins, 2000), Construction and Repair (Chatzikokolakis et al., 2004), Ant Colony Optimization (Blum, 2005) and Particle Swarm Optimization (Sha and Hsu, 2008).

Prins (2000) presents several specialized OSP genetic algorithms with two key features: a population in which each individual has a distinct makespan, and a special procedure which reorders every new chromosome.

Chatzikokolakis et al. (2004) proposed a general repair operator based on local search techniques with a general cost function for evaluating partial assignments. Experimental results improved many best-known solutions of the Guéret-Prins instances.

The basic component of Ant Colony Optimization (ACO) is a probabilistic solution construction mechanism. Because of its constructive nature, it can be regarded as a tree search method. Based on this observation, Blum (2005) hybridizes the solution construction mechanism with beam search. Beam search algorithms are incomplete derivatives of branch-andbound algorithms. It is an approximate method where a partial assignment is only extended in a restricted number of ways (this limit is called the beam width). The approach improved on the results obtained by the current best standard ACO algorithms.

Particle Swarm Optimization (PSO) is a population-based optimization algorithm, where each particle is an individual solution, and the swarm is composed of many particles. Sha and Hsu (2008) modify the representation of particle position, particle movement, and particle velocity to better fit to the OSP. They obtained many optimal solutions to the benchmark problems. 
Finally, many heuristic methods quickly provide good solutions. Most of them are constructive methods and belong to three main families: priority dispatching rules, matching algorithms (see Guéret, 1997), and insertion and appending procedures combined with beam search (see Bräsel et al., 1993).

In this paper, we investigate the enhancements of top-down algorithms for Open-Shop problems as it is important, in practice, to quickly provide good solutions. Our approach relies on the use of strong propagation mechanisms of the unary resource and temporal constraint network constraints but also reasoning dedicated to the minimization of the makespan such as the forbidden intervals method. Our main contribution is to show that randomization and restart strategies combined with strong propagation and scheduling heuristics can lead to a very efficient approach for solving Open-Shop problems. The proposed solving technique outperforms other approaches published so far on a wide range of benchmarks.

This paper is organized as follows. Section 2 introduces our constraint model and Section 3 describes techniques that enhance the search of our top-down algorithm. Finally, Section 4 presents the experimental results we obtained and investigates the effect of each component of the algorithm as well as the comparison against other approaches.

\section{The Constraint Programming Model}

Constraint programming techniques have been widely used to solve scheduling problems. A Constraint Satisfaction Problem (CSP) consists of a set $V$ of variables defined by a corresponding set of possible values (the domains $D$ ) and a set $C$ of constraints. A solution of the problem is an assignment of a value to each variable such that all constraints are simultaneously satisfied. Constraints are handled through a propagation mechanism which allows the reduction of the domains of variables and the pruning of the search tree. The propagation mechanism coupled with a backtracking scheme allows the search space to be explored in a complete way. Scheduling is probably one of the most successful areas for CP thanks to specialized global constraints, which allow modelling resource limitations and temporal constraints.

Constraint programming models in scheduling usually represent a non-preemptive task $T_{i j}$ by a triplet of non-negative integer variables $\left(s_{i j}, p_{i j}, e_{i j}\right)$ denoting the start, processing time and end of the task such that $s_{i j}+p_{i j}=e_{i j}$. In Open-Shop problems, the duration $p_{i j}$ is known in advance and is a constant. The head of a task $e t_{i j}=\inf \left(s_{i j}\right)$, denotes its earliest possible starting time, whereas its tail $l c t_{i j}=\sup \left(e_{i j}\right)$ is its latest completion time.

In this section, we present our constraint programming model to tackle Open-Shop problems. First, we present the unary resource global constraint which models the fact that a single machine or job can be processed at any given time. Then, we explain how precedence constraints are tackled in the decision and propagation process. We also state additional dedicated constraints such as forbidden intervals and symmetry breaking constraints. Finally, various branching schemes in constraint-based scheduling are detailed. 


\subsection{Unary Resource}

A unary resource constraint, also called disjunctive, models a resource of unit capacity. The constraint holds if all the tasks of a collection that have a duration strictly greater than 0 do not overlap. One unary resource constraint is stated for each job and each machine. Let $T$ denote a set of tasks sharing a unary resource and $\Omega$ denote a subset of $T$. We consider the three following propagation rules:

Not First/Not Last: This rule determines if task $i$ cannot be scheduled after or before a set of tasks $\Omega$. In other words, it implies that $i$ cannot be last or first in the set $\Omega \cup\{i\}$. In that case, at least one task from the set must be scheduled after (resp. before) activity $i$ and the tail (resp. head) of $i$ can be updated accordingly.

Edge Finding: This filtering technique determines that some task must be executed first or last in a set $\Omega$. It is the counterpart of Not First/Not Last.

Detectable Precedence: A precedence $i \prec j$ (see Section 2.2) is called detectable if it can be discovered only by comparing the time bounds of its two tasks. Heads and tails of each task can then be updated more accurately by the knowledge of all its predecessors or successors.

Several propagation algorithms (Carlier and Pinson, 1994; Caseau and Laburthe, 1995; Baptiste and Le Pape, 1996; Vilím, 2004) exist for these rules and the best of them have a complexity of $O(n \log (n))$. These rules rely on the computation of the Earliest Completion Time $\left(E C T_{\Omega}\right)$ of a set of tasks $\Omega \subseteq T$. By denoting est $t_{\Omega}=\min _{T_{i j} \in \Omega}\left\{\right.$ est $\left._{i j}\right\}$, its earliest completion time is given by: $E C T_{\Omega}=\max \left\{e t_{\Omega^{\prime}}+\sum_{\Omega^{\prime}} p_{i j}, \Omega^{\prime} \subseteq \Omega\right\}$. We chose the implementation proposed by Vilim (2004) that relies on two efficient data structures: $\Theta$-tree and $\Theta$ - $\Lambda$-tree. These structures are based on a balanced binary tree and allow a quick computation of $E C T_{\Omega}$, especially at each addition or removal of a task in the set.

We also take advantage of the computation of $E C T_{\Omega}$ to estimate a lower bound of the makespan. In fact, the earliest completion time of a machine $M_{i}$ (resp. a job $J_{j}$ ) is estimated by the value of $E C T_{M_{i}}$ (resp. $E C T_{J_{j}}$ ). Thereby, the makespan is greater than the maximum of the earliest completion times among all resources (jobs and machines).

\subsection{Temporal Constraints}

This section deals with the problem of managing quantitative temporal networks without disjunctive constraints. Of course, temporal constraints could be handled by simply adding the corresponding elementary constraints to the solver and by propagating them independently. But, some efficient procedures are dedicated to this problem, known as the Simple Temporal Problem (see Dechter, 2003). This problem involves a set of temporal integer variables $\left\{X_{1}, \ldots, X_{n}\right\}$ and a set of temporal constraints $\left\{a_{i j} \leq X_{j}-X_{i} \leq b_{i j}\right\}$, where $b_{i j} \geq a_{i j} \geq 0$. Cesta and Oddi (1996) proposed algorithms to manage temporal information that: (a) allow dynamic changes of the constraint set for both posting and retraction (b) exploit the temporal constraint network for incremental propagation and cycle detection.

Let $T_{i j} \prec T_{k l}$ denote a precedence constraint, i.e. a temporal constraint such that $s_{i j}+$ $p_{i j} \leq s_{k l}$. A directed graph $G=(V, E)$ is associated with these constraints where the set 
of nodes $V$ represents the set of tasks and the set of arcs represents the set of precedence constraints. Two fictitious tasks $T_{\text {start }}$ and $T_{\text {end }}$ referring to the starting and ending tasks of the schedule, are added to $V$. An arc is added in $E$ between two tasks $T_{i j}$ and $T_{k l}$, if $T_{i j}$ precedes $T_{k l}\left(T_{i j} \prec T_{k l}\right)$. Initially, the only arcs of $E$ are the ones originating at node $T_{\text {start }}$ or ending at node $T_{\text {end }}$.

The structure efficiently handles arc insertions/removals and is restorable upon backtracking, i.e. it maintains a stack to record when a change is done on the graph. Cycle and transitive arc detections have a constant time complexity as we maintain the transitive closure of $G$. Frigioni et al. (2001) proposed an algorithm for maintaining the transitive closure information in a directed graph which requires $O(n)$ amortized time for a sequence of insertions and deletions. In addition, we also maintain a topological order with the simple and efficient algorithm proposed by Pearce and Kelly (2006). Note that, the transitive closure information reduces the overall complexity to maintain a topological order.

The branching strategy (see Section 2.4) adds arcs between tasks sharing a unary resource until all these pairs are connected by a path. At the end of the search, the makespan $C_{\max }$ of a schedule is the length of a longest path between $T_{\text {start }}$ and $T_{\text {end }}$, i.e. a critical path. The branching strategy exploits the transitive closure to avoid creating cycles in the network or branching on transitive or satisfied precedences. Indeed, the precedence network $G$ is consistent if and only if it does not have any cycle. Then, a precedence can be easily detected when it is inferred by the bounds of the tasks, but it is not necessarily the case for transitive precedences. Since precedences satisfy the triangular inequality, if an arc $\left(T_{i j}, T_{k l}\right)$ is transitive, i.e. $T_{i j}$ and $T_{k l}$ are connected by a path in $E \backslash\left\{\left(T_{i j}, T_{k l}\right)\right\}$, then the precedence $T_{i j} \prec T_{k l}$ can be inferred.

Propagation of a set of precedences can be done in linear time, but a bad ordering of awakes in the propagation loop can lead, in the worst case, to quadratic time before reaching the fixpoint. Indeed, the longest path from $T_{\text {start }}$ to $T_{i j}$, and from $T_{i j}$ to $T_{\text {end }}$ are computed to update the head and tail of $T_{i j}$. Since $G$ is a directed acyclic graph, all longest paths originating from $T_{\text {start }}$ and ending at $T_{\text {end }}$ are computed in a linear time with an incremental version of the Dynamic Bellman algorithm for the Single Source Longest Path problem (Gondran and Minoux, 1984). A topological order is an input of the algorithm and our implementation avoids redundant computations by maintaining a dynamic topological order. At each propagation, the algorithm considers only a subgraph of $G$ inferred from tasks for which heads or tails have changed since the last call. Note that the general algorithm proposed by Cesta and Oddi (1996) has a $O(|V| \times|E|)$ complexity whereas our algorithm is $O(|E|)$.

\subsection{Additional Constraints}

In this section, we introduce additional constraints that improve the propagation by considering makespan minimization and basic symmetries. These redundant constraints are dominance rules that are not mandatory for the model's correctness, but improve its resolution. They can be propagated in constant time during the search contrary to complex lower

bounds or dominance rules used in other branching schemes (Brucker et al., 1999), as for instance the Brucker branching scheme. 
Forbidden Intervals Forbidden intervals are a specialized filtering technique for OSP with minimal makespan. Forbidden intervals are intervals in which in an optimal solution, tasks can neither start nor end. Heads and tails can be strengthened based on this information during search. When the head of a task is in such an interval, it can be increased to the upper bound of the interval. This technique has been proposed by Guéret and Prins (1998) and the computation of forbidden intervals is based on the resolution of $m+n$ Subset Sum Problems. The Subset Sum Problem has an $O(d \times n)$ complexity where $d$ is the capacity of the knapsack, i.e. the maximal makespan (see Kellerer et al., 2004). Since these problems are solved once and for all at the beginning of the search, heads and tails are updated in a constant time.

Symmetry Breaking Many constraint satisfaction problems contain symmetries making many solutions equivalent. Symmetry breaking techniques avoid redundant search effort, by trying to ensure that whenever a partial assignment is shown to be inconsistent, no symmetric assignment is ever tried. A solution of the OSP can be reversed considering the last task of a machine as the first, the second to last task as the second and so on. This symmetric counterpart of any solution is also a solution for the OSP. Once the algorithm has proven that one ordering of the tasks is suboptimal, it is unnecessary to check the reverse ordering. Breaking this symmetry can be done by choosing any task $T_{i j}$ and a priori imposing that it

starts in the first half of the schedule: $s_{i j} \leq\left\lceil\frac{e_{e n d}-p_{i j}}{2}\right\rceil$. Our algorithm selects the task with the longest processing time.

\subsection{Branching Scheme}

Branching strategies in scheduling can be divided into two main families: assigning starting times or fixing precedences. The former leads to n-ary branching schemes whereas the latter yields binary decisions.

In the first family, the most well-known strategy is referred to as setTimes (Le Pape et al., 1994) and is an incomplete branching scheme. However, SetTimes is complete in many specific applications including shop problems. At each node, it selects a task from a set of unscheduled and selectable tasks, creates a choice point and schedules the selected task at its earliest starting time. Upon backtracking, it labels the task that was scheduled at the considered choice point as not selectable, as long as its earliest start has not changed.

The second family consists of fixing precedences between tasks. The n-ary branching of Brucker et al. (1997) (denoted as Block) is based on the computation of one heuristic solution at each node to decide which precedences to enforce. The tasks along the critical path of this heuristic solution are selected and precedences are stated to question the current critical path. This branching scheme can fix many precedences simultaneously while remaining complete.

Beck et al. (1997) proposed a simpler binary branching scheme (denoted as Profile) where two critical tasks sharing the same unary resource are ordered. The individual demand is (probabilistically) the amount of a resource required by the activity at time $t$. To estimate contention, the individual demands of each task are aggregated for each resource by summing the individual demand curves for that resource. This aggregate demand curve is used as a measure of the contention for the resource over time. At each node, the resource and 
the time point with the maximum contention are identified, then a pair of tasks that rely most on this resource at this time point is selected (it is also ensured that these two tasks are not already connected by a path of temporal constraints). Once the pair of tasks has been chosen, the order of the precedence has to be decided. This can be done using the randomized value ordering heuristics known as Centroid (Beck et al., 1997). The centroid is a real deterministic function of the domain and is computed for the two critical tasks. The centroid of a task is the point that divides its demand curve equally. We commit the precedence which preserves the ordering of the centroids of the two tasks. If the centroids are at the same position, a random ordering is chosen.

More recently, Laborie (2005) proposed a branching scheme for general cumulative scheduling relying on the notion of minimal critical sets (MCS). In disjunctive scheduling context, MCSs are pairs of activities conflicting for the same unary resource. At each node, the branching consists of (a) selecting a MCS according to an estimation of the related reduction of the search space, (b) applying a simplification procedure on each MCS, and (c) branching on its possible precedences in the children nodes until no MCS remains.

An example of each branching is given in Figure 2 to illustrate the various shapes of the search tree. We implemented the Profile branching scheme because the use of randomized binary decisions eases the integration of techniques detailed below.

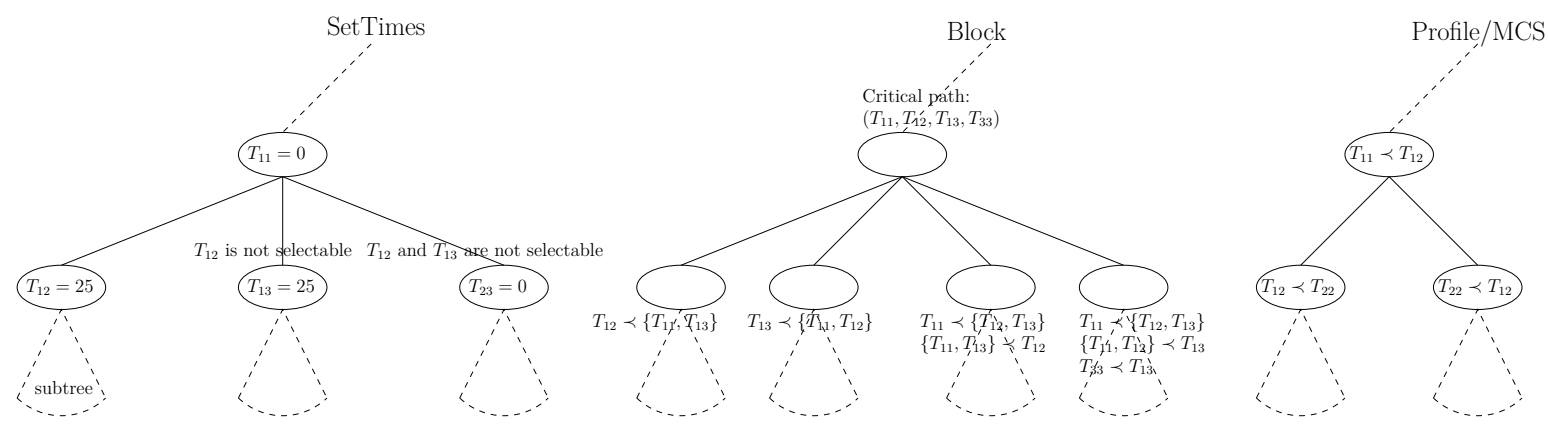

Figure 2: The shape of the search tree for different branching schemes. From left to right, SetTimes, Block and Profile/MCS branching schemes.

\section{Solving the Open-Shop Problem}

Using the model presented in Section 2, we now can solve the Open-Shop problem by using a top-down search. This section describes the enhancements, with scheduling heuristics, and randomization and restart of our constraint model based on the propagation techniques of the unary resource, precedence network and additional constraints. The branching is conducted by adding precedences using the Profile heuristics with randomized Centroid. Preliminary experiments reveal two main drawbacks. First, propagation techniques are very effective once a tight upper bound is known, but only slow down search otherwise. Second, the slightly randomized version of Centroid shows a large variance both in solving time and in quality of the first solutions found. Such a behavior suggests that decisions taken early in the search tree are never questioned again leading to an important thrashing phenomenon (the same failure can be rediscovered several times). 
We propose to apply a randomized constructive heuristic (without propagation) which initializes the upper bound so that the selection and propagation of initial choices is improved at the beginning of the complete search. Furthermore, we propose a restarting strategy enhanced with nogood recording at each restart to prevent exploring the same part of the search space again. Note that these two techniques can be used in any top-down approach and with any branching scheme, although recording nogoods is made easier by using binary decisions. We now give a detailed presentation of the overall approach and analyze its parameters.

\subsection{Main Procedure}

Figure 3 summarizes a general algorithm for solving Open-Shop problems: the Randomized and Restarts Constraint Programming algorithm for Open-Shop problem (RRCP). Possible values of the relevant parameters are indicated in blocks 1 and 7 , and are discussed in Section 4.1. The top-down algorithm starts with a heuristic (blocks 1-2) and continues, if needed, with a complete search (blocks 3 to 9) which incrementally improves the best solution found so far. The heuristic computes an initial solution and provides the initial upper bound $C_{\max }^{U B}$. We designed a simple randomized heuristic named CROSH, presented in detail in Section 3.2. Then, an optimality test is applied (block 2) before going any further and starting the search. In block 3, the CP model is created (see Section 2) and various components are initialized.

The generic loop of the algorithm is contained between blocks 4 and 9. After propagation and domain reduction (block 4), we may have either reached a solution, a contradiction, or neither of those two cases. If a solution is found, it is recorded and the new upper bound on the makespan is used to add a constraint as a dynamic cut (block 5) that is propagated upon backtracking (block 6). If a failure is detected and all branches of the root node have been fathomed, then optimality of the last solution found is proven and the algorithm terminates. Otherwise, the algorithm backtracks (block 6). Then, at that point, a new branching step is needed but before that, we examine the possibility of restarting.

Two different policies for restarting (Luby, Walsh) have been analyzed in our study as shown on block 7 and discussed in detail in Section 3.3. In the case of restarts, we extract nogoods (block 8) from the last branch of the search tree to avoid redundant work from one restart to the next and keep track of the subproblems already proven to be suboptimal or infeasible. A nogood is defined here by the current upper bound and the set of branching decisions (precedences). Note that, the algorithm does not restart before a backtrack to avoid side-effects of recording truncated nogoods or restarting at the root node. If no restart is performed, then a search is undertaken using the Profile branching scheme in block 9 (see Section 2.4). Branching divides the main problem into a set of disjoint subproblems by temporarily adding a precedence.

\subsection{Initial Solution}

As mentioned above, propagation techniques are very costly and only useful when applied with a tight upper bound. Similarly, the branching technique is very sensitive to the quality 


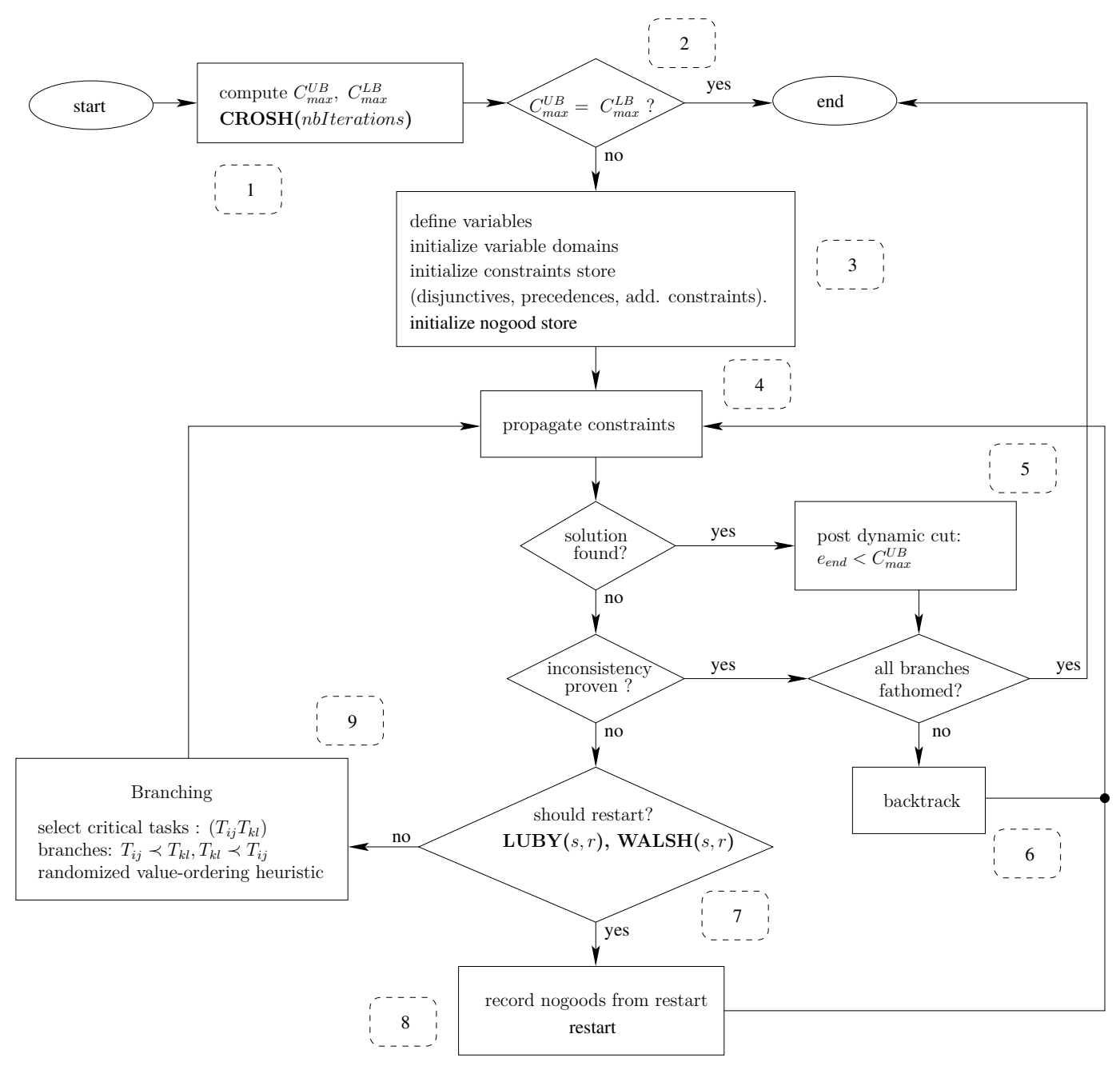

Figure 3: General outline of RRCP. Ellipses are initial and final states. Rectangles are procedures or actions. Diamonds are if-else conditions. Dashed rectangles are labels.

of the upper bound, as it relies on the demand curve of the resources. It is thus important to provide a good upper bound at the root node in a short period.

We designed a Constructive Randomized Open-Shop Heuristics (CROSH) by combining a randomization step with Priority Dispatching Rule (PDR) methods. Aside from being generic and simple to implement, CROSH yields good results experimentally (see Section 4.2). PDR methods are classical methods to construct a non-delay schedule by repeatedly appending tasks to a partial schedule (Kolisch, 1996). A schedule is called a non-delay if no machine is left idle, provided that it is possible to process some job. Starting with an empty schedule, tasks are appended as follows: (a) determine the minimal head $t_{0}$ of all unscheduled operations (at time $t_{0}$, there exists both a free machine and an available job), (b) among all available tasks, choose one according to a dispatching rule. The first iteration applies the Longest Processing Time (LPT) rule. Following iterations uniformly select a random task at step (b) instead of following a dispatching rule. The only parameter of CROSH is its number of iterations. The complexity of one iteration is $O\left(m^{2} \times n^{2}\right)$. 


\subsection{Restart Strategy}

Restart policies are based on the following observation: the longer a backtracking search algorithm runs without finding a solution, the more likely it is that the algorithm is exploring a barren part of the search space. Initial choices made by the branching are both the least informed and the most important, as they lead to the largest subtrees and the search can hardly recover from early mistakes. This can lead to thrashing situations where failures are due to a small subset of early choices but discovered much deeper in the tree over and over again.

To address this issue, shaving and intelligent backtracking techniques have been widely studied (Guéret et al., 2000; Dorndorf et al., 2001; Laborie, 2005). Shaving tries to assign a value to a variable and applies a consistency filtering algorithm. If an inconsistency is found, then the value can be safely removed from the domain of the variable. An intelligent backtracking algorithm tries to compensate for the early mistakes of the branching by analyzing failures and identifying the choices responsible for the current dead end.

We implemented restart strategies combined with randomization which is another way to get rid of thrashing and bad initial choices. Such techniques diversify the search and require less computation at each node than shaving or intelligent backtracking but explore more nodes. Preliminary experiments have shown that shaving techniques are not useful in our implementation. Note that, intelligent backtracking has not been experimented because it requires the explanation of all domain changes as well as a deep integration into the search algorithm.

Universal Restart Strategy Let $A(x)$ be a randomized algorithm of the Las Vegas type, which means that, on any input $x$, the output of $A$ is always correct but its running time $T_{A}(x)$ is a random variable. A universal restart strategy determines the length of any run for all distributions on running time.

If the only feasible observation is the length of a run and there is no knowledge of the runtime distribution of the solver on the given instance, Luby et al. (1993) showed that the universal schedule of cutoff values of the form $(1,1,2,1,1,2,4,1,1,2,1,1,2,4,8, \ldots)$ gives an expected time to solution that is within a log factor of that given by the best fixed cutoff, and that no universal schedule is better by more than a constant factor. The two parameters that we consider are a scale factor $s$ and a geometric factor $r$. The scale factor is the base

cutoff in a restart strategy. By denoting $\lambda_{k}=\frac{r^{k}-1}{r-1}$, the $i$-th term of the sequence is $(s=1$ and $r=2$ is the previous example):

$$
\begin{array}{r}
\forall i>0, \quad t_{i}= \begin{cases}s r^{k-1} & \text { if } i=\lambda_{k} \\
t_{i-\lambda_{k-1}} & \text { if } \lambda_{k-1}<i<\lambda_{k}\end{cases} \\
s=2 \text { and } r=3 \Rightarrow 2,2,2,6,2,2,2,6,2,2,2,6,18, \ldots
\end{array}
$$

Walsh (1999) suggested another universal strategy of the form $s, s r, s r^{2}, s r^{3}, \ldots$ growing exponentially, contrary to the Luby strategy which grows linearly.

Wu and van Beek (2007) demonstrated the pitfalls of non-universal strategies both analytically and empirically, and showed that parametrization of the strategies improves performance while retaining any optimality and worst-case guarantees. As restarting seems a 
key component of those problems, we evaluated the effects of scale and geometric factors to identify good restart strategies.

Nogood Recording from Restarts Our branching scheme is only randomized when ordering two tasks to state a precedence, and even in this case, the randomization only takes place when Centroid is unable to identify a good order. This slight randomization of the search is enough, as mentioned previously, to observe a large variance in solution quality. Sometimes, only a few random choices are made and the same search tree is likely to be explored from one restart to another. We apply a simple nogood recording technique similar to Lecoutre et al. (2007) to compensate for this drawback.

In our context, a nogood is defined by the current upper bound $u b$ and corresponds to a set of precedences $P$, such that all solutions satisfying $P$ have a makespan greater than $u b$. The same set $P$ of precedences can be met from one restart to another. Recording $P$ can avoid redundant work and provide more diversification across the restarts. We record nogoods only when the search is about to restart (block 8 of Figure 3). At this point we record all the nogoods representing the subtrees proven suboptimal following the idea of Lecoutre et al.. All the work accomplished during this step is therefore recorded and the same part of the search tree will therefore not be explored in latter runs. Since a nogood is extracted from each negative decision of the last branch in a binary branching tree, only a linear number of nogoods, with respect to the number of precedences, is recorded at each restart.

Nogoods are propagated individually in Lecoutre et al. using watch literals techniques. We implemented the nogood store as a global constraint that achieves unit propagation on the nogoods. Our implementation remains naive and could be improved based on watch literals techniques. However, the number of nogoods remains quite small in practice as they are only recorded at each restart and nogood propagation didn't seem to be a bottleneck for efficiency in our approach. Furthermore, we remove nogoods that are subsumed by others when adding all the nogoods coming from a new restart.

\section{Computational Results}

Three sets of OSP benchmark instances are available in the literature. The first set consists of 60 problem instances provided by Taillard (1993) (denoted by tai*) ranging from 16 operations ( 4 jobs and 4 machines) to 400 operations (20 jobs and 20 machines). This set of instances is considered easy because no optimality proof is needed, i.e. the optimal makespan is equal to the lower bound. Brucker et al. (1997) proposed 52 difficult instances (denoted by $\left.\mathrm{j}^{*}\right)$ from 3 jobs and 3 machines to 8 jobs and 8 machines. Finally, the last set consists of 80 benchmark instances provided by Guéret and Prins (1999) (denoted by GP*). The size of these instances ranges from 3 jobs and 3 machines to 10 jobs and 10 machines and the optimal makespan is always strictly greater than the lower bound. Note that, the lower

bound $C_{\max }^{L B}$ is always equal to 1000 on Brucker and Guéret-Prins benchmark instances.

We perform several sets of experiments in order to: (a) configure the parameters (Section 4.1); (b) study the impact of various components of the algorithm (Section 4.2); (c) compare RRCP with state-of-the-art methods (Section 4.3). Two sets of independent experiments were designed to achieve step (a) in a reasonable amount of time. Using the best parame- 
ters, we conducted a main set of experiments in order to complete steps (b) and (c). The algorithm applied on the complete benchmark uses CROSH/LPT in a first step, and applies, or not, a Luby/Walsh restarting policy with/without nogood recording. As the algorithm is randomized, 20 runs were performed for each instance without a time limit. Solving time includes the computation of the initial upper bound.

Our implementation is based on the Choco solver (Java) extended with scheduling objects (tasks, resources, temporal constraints, and branching) and restarting policies with nogood recording. Given that these features have been integrated in the latest releases $(\geq 2.0 .0)$, our algorithm is easily reproducible. An additional package provides scheduling heuristics, builds the model, and configures the solver.

All experiments were performed on a cluster of Linux machines, each node with 1 GB of RAM and a AMD 2.2 GHz processor.

\subsection{Setting the Parameters of the Algorithm}

The parameters of our algorithm RRCP are presented in Section 3. An experimental study to justify the choices made in the final set up of the algorithm is reported here.

\subsubsection{Initial Solution}

In this section, we discuss how to fix the number of iterations of CROSH outlined in block 1 of Figure 3. This set of experiments aims to determine the balance between the time spent with the heuristics and the quality of the provided upper bound. Ideally, we wish to stop the heuristic phase as soon as the CP search can improve the solution faster.

Therefore, we discretized the number of iterations into orders of magnitude 1, 10, 100, $1000,5000,10000,25000$. The maximum number of iterations was set to 25000 because a timeout of 30 seconds was reached after 25000 iterations for large instances $(15 \times 15,20 \times 20)$. In all instances, we applied the version of our algorithm that uses CROSH in a first step, and does not include a restarting strategy. Twenty runs were performed for each instance with a time limit of 180 seconds.

We deduce an estimated number of iterations for each problem's size from the percentage of solved instances and the average solving time. The instances until the size $6 \times 6$ are easily solved by the constraint model and the number of iterations is fixed to 1000 . Then, the number of iterations is fixed to 10000 iterations until the size $9 \times 9$, and to 25000 iterations otherwise. The time limit of CROSH is fixed to 20 seconds.

\subsubsection{Restart Policy Parameters}

This section addresses how the restart policy parameters outlined in block 7 of algorithm 3 (scaling and geometric factors) are fixed. We selected a set of 23 instances from the size $6 \times 6$ to $20 \times 20\left(8 \mathrm{GP}^{*}, 9 \mathrm{j}^{*}, 6 \mathrm{tai}^{*}\right)$ with different runtime distributions to identify good values for the parameters. We report the effects of the parameters on the efficiency of the restart policy measured by the number of problems solved to optimality as proposed by Wu and van Beek (2007). The scale factor $s$ is discretized into orders of magnitude $10^{-2}, 10^{-1} \ldots, 10^{2}$ and the geometric factor $r$ into 2, 3, ., 10 for Luby and 1.1, 1.2, ., 2 for Walsh. Then, the scale factor is multiplied by the number of tasks $n \times m$ to take into account the size of 
the problem. Indeed, the scale factor is often related to the size, depth and width, of the search tree. The multiplication balances the number of restarts for different problem's sizes. Twenty runs were performed on each instance of the set, with a time limit of 180 seconds and an initial upper bound given by LPT (all runs start with the same upper bound).

The best parameter settings were then estimated by choosing the values that maximized the expected number of instances solved. Ties were broken by considering the average amount of time needed to solve an instance. Table 1 shows the results of the experiments for the two restart policies with nogood recording. The percentage of solved instances, the

\begin{tabular}{|c|c|c|c|c|c|c|c|c|}
\hline & \multicolumn{4}{|c|}{ Luby } & \multicolumn{4}{|c|}{ Walsh } \\
\hline & $(s, r)$. & $\%$ & $\bar{t}$ & $\bar{n}$ & $(s, r)$ & $\%$ & $\bar{t}$ & $\bar{n}$ \\
\hline Best & $(1,3)$ & 82.6 & 43.1 & 10055 & $(1,1.1)$ & 82.6 & 43.0 & 9863 \\
\hline Average & $(*, *)$ & 75.5 & 58.6 & 16738 & $(*, *)$ & 76.2 & 54.6 & 11359 \\
\hline Acceptable & $(1, *)$ & 82.0 & 44.5 & 10347 & $(0.1, *)$ & 80.2 & 48.0 & 10973 \\
\hline NotAcceptable & $(0.01, *)$ & 72.9 & 67.4 & 31676 & $(100, *)$ & 70.5 & 67.1 & 12600 \\
\hline
\end{tabular}

Table 1: Identifying good parameters for the restart policies with nogood recording.

average amount of time and number of nodes visited during search are given for different sets of parameters. The symbol* represents all possible values for a parameter. The Best line reports results of the two pairs of selected parameters. The Average line reports average results over all parameters settings. The Acceptable and NotAcceptable lines respectively report average results for scale factors leading to significant performance improvement and degradation. As expected, estimating good parameters (Best and Acceptable) settings can give quite reasonable performance improvements over unparametrized universal strategies (Average, NotAcceptable). Many parameter settings obtained similar results where their quality was highly depending upon the value of the scale factor (Acceptable and NotAcceptable). Using their best parameters, the equivalence of Luby and Wash policies with nogood recording is experimentally shown in Section 4.2.2. Therefore, other sections report only the results obtained with Luby policy with nogood recording.

\subsection{Sensibility Analysis}

We report here an experimental study on the influence of the techniques introduced in Section 3 and justify their use experimentally. We only report the results of instances with size greater than $6 \times 6$ because the short solution times of small instances are not significant. Let recall that 20 runs were performed for each instance as the algorithm is randomized.

\subsubsection{Initial Solution}

In this section, we study the impact of CROSH on different benchmarks, and compare CROSH versus LPT, i.e. its first iteration. Preliminary analysis, not detailed here, showed that CROSH can execute 10000 iterations in less than 1 second for instances of size up to $9 \times 9$ (inclusive) and 25000 iterations in less than 5 seconds for the size $10 \times 10$. For large Taillard instances, "easy" instances are most often solved optimally within a few seconds, reaching 20 seconds to achieve 25000 iterations in a few cases. 
Let $C_{\max }^{U B}$ be the initial upper bound given by CROSH. The optimality gap is the ratio of the difference between this upper bound and the optimal makespan, on the optimal makespan: $\left(C_{\max }^{U B}-C_{\max }\right) \div C_{\max }$. The left graph of Figure 4 illustrates the relation between the average optimality gap of CROSH and the solving time of RRCP (given on the horizontal axis). Each point represents one instance and its $x$ coordinate is the average solving time of RRCP (logarithmic scale), whereas its $y$ coordinate is the average optimality gap of CROSH. The initial solution quality is satisfactory because the gap never exceeds $4 \%$ and is optimal or near-optimal in many instances. The solving time of RRCP is not clearly related to the optimality gap, especially for the Taillard benchmark. However, gaps increase on hardest Guéret-Prins and Brucker instances, whereas they decrease on largest Taillard instances. Further analysis, not detailed here, showed that CROSH gives the optimum at least once for 28 of 40 Taillard instances, whereas it happened respectively for 6 of 40 Guéret-Prins instances, and for 3 of 26 Brucker instances. Furthermore, all runs gave the optimum for 10 large Taillard instances $(15 \times 15,20 \times 20)$.
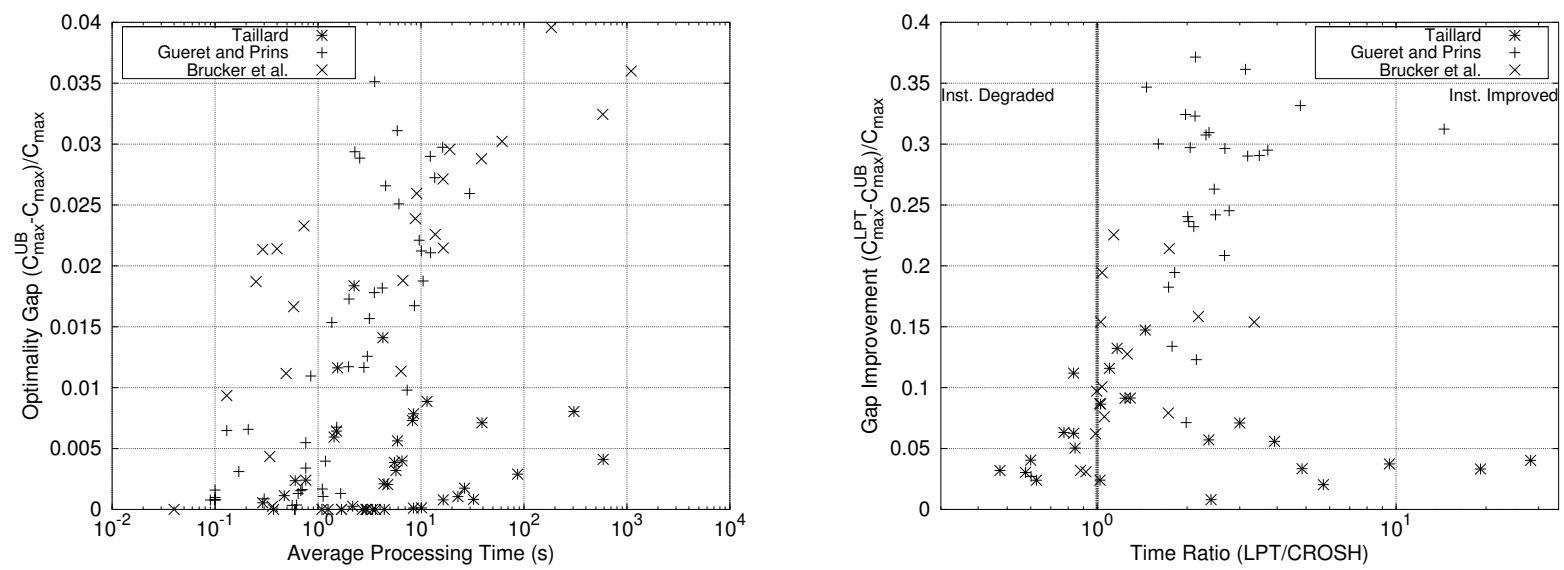

Figure 4: Optimality gap for the initial upper bound and impact of CROSH on RRCP.

Let $C_{\max }^{L P T} \geq C_{\max }^{U B}$ be the upper bound given by LPT, the first iteration of CROSH. The gap improvement of CROSH over LPT is estimated by calculating the ratio of the difference between the initial bounds given by LPT and CROSH over the optimal makespan: $\left(C_{\max }^{L P T}-\right.$ $\left.C_{\max }^{U B}\right) \div C_{\max }$. The average gap improvement is only $5.7 \%$ on Taillard benchmarks because LPT provides tight bounds on these instances. But, it grows until respectively $10.6 \%$ and $25.8 \%$ for Brucker and Guéret-Prins benchmarks because LPT obtains weaker results.

The right graph of Figure 4 shows the impact of CROSH on our algorithm. Each point represents one instance solved with a Luby restarting policy with nogood recording. Its $x$ coordinate is the ratio (logarithmic scale) of the average solving time using an initial bound given by LPT over the average solving time using an initial bound given by CROSH whereas its $y$ coordinate is the average gap improvement. The 67 instances strictly greater than size $6 \times 6$ and solved at least once with an average time between 2 seconds and 1800 seconds are considered to plot this graph. All points located on the right of the line $\left(x=1\left(=10^{0}\right)\right)$ are instances improved by the use of CROSH. Solution time improvement seems related to the gap improvement with the exception of Taillard instances. In spite of similar gap improvements, 
some Taillard instances are solved more than 10 times faster using CROSH whereas a few instances located on the left of $(x=1)$ are degraded.

\subsubsection{Restart Strategy}

In this section, we study the impact of restarting strategies and demonstrate experimentally the equivalence between Luby and Walsh strategies with nogood recording in context of OSP. Using the best parameters for Luby and Walsh given in Table 1, we show the interest of restarting strategies as well as the effect of enhancing them with nogood recording on the two graphs of Figure 5. The 61 instances strictly greater than size $6 \times 6$ and solved with an average time between 2 seconds and 1800 seconds are considered to plot those graphs. The initial upper bound is given by CROSH.

The left graph analyzes the effect of the restarting strategies. Each point represents one instance and its $x$ coordinate is the ratio of the solving time without restarts over the solving time with restarts, whereas its $y$ coordinate is the ratio of the number of nodes without restarts over the number of nodes with restarts. Notice also that both scales are logarithmic. All points located above and on the right of the point $(1,1)$ are instances improved by the use of restarts (top-right quadrant). On the contrary, all points located below and on the left of the point $(1,1)$ are instances degraded by the use of restarts (bottom-left quadrant). As expected, all points are around the diagonal as the number of nodes is roughly proportional to the time (top-left and bottom-right quadrants are empty). Restarting globally improves the solution and some instances are even solved approximately 100 times faster using restarts. However, the performances over a number of instances located below $(1,1)$ are degraded.
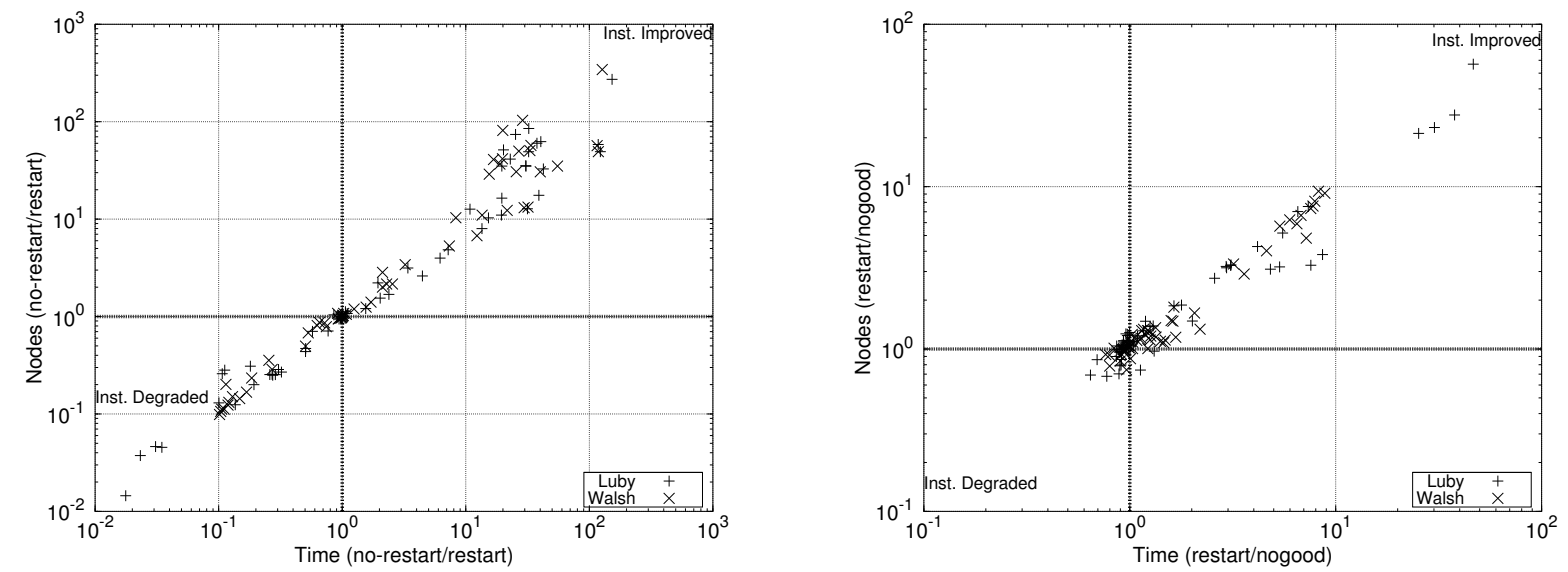

Figure 5: Impact of restart policies (left graph) and nogood recording over restart policies (right graph).

Similarly, the right graph shows the gain offered by nogood recording over the use of restart policy (the coordinates of each point present the ratio of time and nodes of the restarting strategy alone over the restarting strategy with nogood recording). One can see that nogood recording improves the restarting policies by a factor between 1 and 10 in the large majority of instances.

Finally, when combining restarting policy and nogood recording, we obtain the results plotted 
in the left graph of Figure 6 (the coordinates of each point present the ratio of time and nodes without restarts over the restarting strategy with nogood recording). Thus all the negative results of the restarting policy of Figure 5 have been eliminated, while keeping the positive effects of the restarts.

We have shown here that restarting alone can greatly improve the solution of Open-Shop problems but lacks robustness. Restarting basically helps to find good upper bounds quickly, but once those are known, longer runs are eventually needed to prove optimality. The balance between restarting quickly to improve the upper bound and searching more to prove optimality is difficult to achieve. Enhancing the restarting policy with nogood recording compensates for this drawback and improves the resolution significantly, as shown by the left graph of Figure 6.
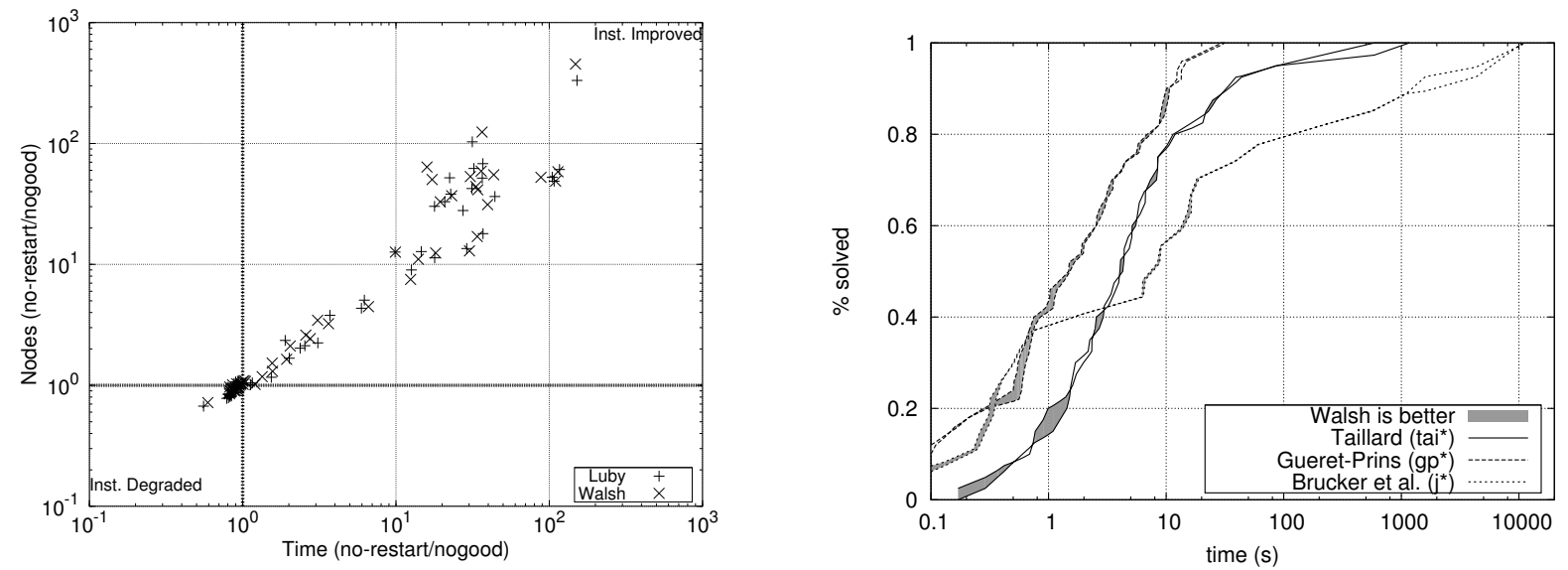

Figure 6: Impact of restart policies combined with nogood recording (left graph) and equivalence of restart policies with nogood recording (right graph).

Finally, the right graph of Figure 6 shows that Luby and Walsh policies with nogood recording are equivalent when using their best parameters. For each benchmark, and for each policy, the percentage of solved instances is drawn as a function of solution time. The two curves belonging to the same benchmark are represented with the same line pattern and the area between them is filled in grey if the Wash policy performs better than the Luby's policy, and is left blank otherwise. In our context, Luby and Walsh policies are equivalent as their two curves nearly coincide for each benchmark. Furthermore, the graph indicates an order of difficulty between the benchmarks: Guéret-Prins $\prec$ Taillard $\prec$ Brucker.

Table 2 summarizes solution times (hour:minute) and numbers of nodes (millions of nodes) obtained on the three hardest instances (solving times are greater than 1800 seconds). RRCP uses the initial upper bound given by CROSH. We first note that the strategy without restart performs better as it is necessary to explore many nodes to provide a proof of optimality. Nogood recording is a critical issue for restart strategies on these instances, as it cuts the runtime by two thirds. Without nogood recording, all runs between the discovery of the optimum and the last are useless since proven infeasibilities are forgotten from one restart to another. Despite of nogood recording, the significant number of restarts, especially with the Luby policy, tends to increase slightly the solution time as the selection and 
propagation of initial choices are repeatedly performed. The Walsh strategy with nogood recording yields slightly better solution time than the Luby's policy. However, its efficiency heavily depends on nogood recording as it performs longer runs.

\begin{tabular}{|c|c|c|c|c|c|c|c|c|c|c|c|}
\hline & & & & \multicolumn{4}{|c|}{ Restart } & \multicolumn{4}{|c|}{ Nogood Recording } \\
\hline \multicolumn{2}{|c|}{ Instance } & \multicolumn{2}{|c|}{ No Restart } & \multicolumn{2}{|c|}{ Luby } & \multicolumn{2}{|c|}{ Walsh } & \multicolumn{2}{|c|}{ Luby } & \multicolumn{2}{|c|}{ Walsh } \\
\hline Name & Opt & $\bar{t}$ & $\bar{n}$ & $\bar{t}$ & $\bar{n}$ & $\bar{t}$ & $\bar{n}$ & $\bar{t}$ & $\bar{n}$ & $\bar{t}$ & $\bar{n}$ \\
\hline j7-per0-0 & 1048 & $1: 43$ & 1.21 & $5: 56$ & 4.66 & $18: 10$ & 12.76 & $2: 10$ & 1.57 & $2: 03$ & 1.25 \\
\hline j8-per0-1 & 1039 & $2: 13$ & 1.16 & 10:22 & 5.95 & $23: 12$ & 12.29 & 3:07 & 1.65 & $3: 00$ & 1.38 \\
\hline j8-per10-2 & 1002 & 1:03 & 0.56 & $8: 46$ & 5.11 & $8: 50$ & 4.72 & 1:17 & 0.68 & $1: 13$ & 0.57 \\
\hline
\end{tabular}

Table 2: The average solution time $\bar{t}$ (hour:minute) and number of nodes $\bar{n}$ (millions of nodes) for the given alternatives applied to the three hardest instances.

\subsubsection{Robustness}

Lastly, we analyzed the robustness of RRCP for the Luby restart policy with nogood recording and the initial upper bound given by CROSH. Robustness refers, in our case, to the sensitivity to the initial upper bound and to the randomized decision process, from one run of RRCP to another. For each instance, we compute the ratio of the standard deviation on the average runtime: $\operatorname{std}(\bar{t}) \div \bar{t}$. Then, we compute the average ratio for each benchmark. The Taillard benchmark gives the highest average ratio equal to $62 \%$ because no optimality proof is needed. Solution times largely differ according to the optimality of the initial upper bound from one execution to another. The instance is closed without branching if the initial bound is optimal, otherwise starting a complete search leads to an increase in solution time. At the contrary, our algorithm is more robust on Guéret-Prins and Brucker benchmarks with average ratios of $16 \%$ and $9 \%$. Indeed, it spends more time to prove optimality than to reach an optimal solution because the proof requires the exploration of many nodes.

\subsection{Comparison to Other Approaches}

In this section, we compare results of our algorithm without time limit, using the Luby restarting policy with nogood recording and the initial bound given by CROSH, to other approaches. Tables 3, 4 and 5 summarize the results of different approaches on the Taillard, Brucker and Guéret-Prins benchmarks. The tables include the best results obtained by the genetic algorithm (GA-Pri - Prins, 2000), the ant colony algorithm (ACO-Blu - Blum, 2005), the particle swarm algorithm (PSO-Sha - Sha and Hsu, 2008), the branch-and-bound with intelligent backtracking (BB-Gue - Guéret et al., 2000), the branch-and-bound with consistency tests (BB-Do - Dorndorf et al., 2001), and the transformation into SAT of (SAT-Ta Tamura et al., 2006). The papers cited above sometimes report several results obtained with variants of their approach, and we have quoted the best of them in Tables 3, 4 and 5. The column Opt gives the optimal makespan for each instance. The value of a reported objective is in bold when it is equal to the optimal makespan.

We report the objective value after a unique run of GA-Pri and do not report its solving 
time. ACO-Blu and PSO-Sha results were obtained by 20 runs on each problem using PCs with AMD Athlon $1.1 \mathrm{GHz}$ CPU running under Linux, and PCs with AMD Athlon $1.8 \mathrm{GHz}$ running under Windows XP respectively. For each of these approaches, the average objective value (Avg) and average solving time $\bar{t}$ are given. The best objective value (Best) is also given when it is not equal to the optimal makespan for all instances. The best objective value of PSO-Sha is marked with a $\dagger$ if the decoding operator is not hybridized with beam search.

We report only the objective value of BB-Gue as it reached often the limit of 250000 backtracks (about 3 hours of CPU time on a Pentium PC clocked at $133 \mathrm{MHz}$ ).

We report the solving time $t$ of BB-Do, which has been tested on a Pentium II $333 \mathrm{MHz}$ in an MSDOS environment with a time limit of 5 hours, since it identified many optimal solutions. The symbol - indicates that the bottom-up algorithm was stopped before finding a solution, whereas the final upper bound is given in brackets when the top-down algorithm was interrupted.

Solution times of SAT-Ta using Intel Xeon 2.8GHz 4GB memory are reported with the exception of the experiments on j7-per0-0 and j8-per0-1 problems which were done using 10 Mac mini machines (PowerPC G4 1.42GHz 1GB memory) running in parallel and by dividing each problem into 120 subproblems. Optimal solutions were found and proven for both instances within 13 hours (marked with $M$ in Table 4).

(CNR-Cha - Chatzikokolakis et al., 2004) interrupted their search after 120 minutes and only if the time elapsed after the last improvement exceeded 30 minutes. CNR-Cha did not report either solution times, or makespan.

MCS-Lab applied their bottom-up algorithm with a time limit of 5 seconds for each subproblem and ran experiments on a Dell Latitude D600 laptop, $1.4 \mathrm{GHz}$. If the time limit was reached on a given subproblem, then the search was stopped without returning any solution. MCS-Lab did not report solution times but it is possible to estimate an overall runtime for each instance. A bottom-up algorithm solves $C_{\max }-C_{\max }^{L B}$ infeasible subproblems and a single feasible subproblem giving the optimum. The number of subproblems depends on each particular instance despite $C_{\max }^{L B}$ is always equal to 1000 for Brucker and Guéret-Prins benchmarks. MCS-Lab reported that the early iterations were short and that the latter ones become longer during the transition phase. Therefore, our estimation of its runtime, inspired by the dichotomous variant of bottom-up (see Section 1), is equal to $5 \times\left\lceil\log _{2}\left(C_{\max }-C_{\max }^{L B}+1\right)+1\right\rceil$ seconds.

As mentioned before, our experiments were realized on a cluster with Linux machines, each node with $1 \mathrm{~GB}$ of RAM and a AMD $2.2 \mathrm{GHz}$ processor. We report the average solving time $\bar{t}$ and number of nodes $\bar{n}$ of our approach over 20 runs. Note that the comparison of the solving times might not be always significant because of the differences among computational platforms.

Results for the Taillard Instances (Table 3) This set of instances is considered easy since no optimality proof is needed, and it is thus solved efficiently by metaheuristics. For example, only six instances remained unsolved after a unique run of GA-Pri. ACO-Blu and PSO-Sha closed the remaining instances with good solution times even if some runs on several instances led to suboptimal solutions. These failures are not clearly related to the size of the problem. On the contrary, CNR-Cha obtained its weakest results on this 


\begin{tabular}{|c|c|c|c|c|c|c|c|c|c|c|}
\hline \multirow{2}{*}{\multicolumn{2}{|c|}{ Instance }} & \multicolumn{5}{|c|}{ Metaheuristics } & \multicolumn{4}{|c|}{ Exact Algorithms } \\
\hline & & \multirow[t]{2}{*}{ GA-Pri } & \multicolumn{2}{|c|}{ ACO-Blu } & \multicolumn{2}{|c|}{ PSO-Sha } & \multirow{2}{*}{$\frac{\mathrm{BB}-\mathrm{Do}}{t}$} & \multirow{2}{*}{$\frac{\mathrm{SAT}-\mathrm{Ta}}{t}$} & \multicolumn{2}{|c|}{ RRCP } \\
\hline Name & Opt & & Avg & $\bar{t}$ & Avg & $\bar{t}$ & & & $\bar{t}$ & $\bar{n}$ \\
\hline tai_7_7_1 & 435 & 436 & 435 & 2.1 & 435 & 2.9 & 0.4 & 21 & 1.6 & 355 \\
\hline tai_7_7_2 & 443 & 447 & 443 & 19.2 & 443 & 12.2 & 0.9 & 24 & 1.6 & 448 \\
\hline tai_7_7_3 & 468 & 472 & 468 & 16.0 & 468 & 9.2 & 30.9 & 30 & 4.3 & 1159 \\
\hline tai_7_7_4 & 463 & 463 & 463 & 1.7 & 463 & 3.0 & 5.3 & 20 & 1.5 & 478 \\
\hline tai_7_7_5 & 416 & 417 & 416 & 2.3 & 416 & 2.9 & 2.0 & 22 & 0.8 & 157 \\
\hline tai_7_7_6 & 451 & 455 & 451.4 & 24.8 & 451 & 13.5 & 95.8 & 45 & 11.5 & 3945 \\
\hline tai_7_7_7 & 422 & 426 & 422.2 & 23.0 & 422 & 13.6 & 167.7 & 33 & 2.3 & 602 \\
\hline tai_7_7_8 & 424 & 424 & 424 & 1.2 & 424 & 2.3 & 5.0 & 20 & 0.6 & 189 \\
\hline tai_7_7_9 & 458 & 458 & 458 & 1.1 & 458 & 1.3 & 0.8 & 21 & 0.3 & 109 \\
\hline tai_7_7_10 & 398 & 398 & 398 & 1.6 & 398 & 2.8 & 53.2 & 20 & 0.5 & 105 \\
\hline tai_10_10_1 & 637 & 637 & 637.4 & 40.1 & 637 & 9.4 & 30.2 & 98 & 8.3 & 1214 \\
\hline tai_10_10_2 & 588 & 588 & 588 & 3.0 & 588 & 3.5 & 70.6 & 95 & 4.8 & 667 \\
\hline tai_10_10_3 & 598 & 598 & 598 & 27.9 & 598 & 10.1 & 185.5 & 92 & 8.5 & 1162 \\
\hline tai_10_10_4 & 577 & 577 & 577 & 2.6 & 577 & 2.6 & 29.7 & 92 & 2.2 & 264 \\
\hline tai_10_10_5 & 640 & 640 & 640 & 8.6 & 640 & 4.0 & 32.0 & 96 & 6.6 & 830 \\
\hline tai_10_10_6 & 538 & 538 & 538 & 2.6 & 538 & 1.1 & 32.7 & 95 & 0.4 & 0 \\
\hline tai_10_10_7 & 616 & 616 & 616 & 5.2 & 616 & 3.9 & 30.9 & 103 & 4.4 & 403 \\
\hline tai_10_10_8 & 595 & 595 & 595 & 15.0 & 595 & 7.0 & 44.1 & 95 & 6.0 & 633 \\
\hline tai_10_10_9 & 595 & 595 & 595 & 5.1 & 595 & 4.1 & 39.8 & 97 & 5.8 & 541 \\
\hline tai_10_10_10 & 596 & 596 & 596 & 7.5 & 596 & 5.0 & 29.1 & 95 & 5.6 & 541 \\
\hline tai_15_15_1 & 937 & 937 & 937 & 14.3 & 937 & 4.3 & 481.4 & 523 & 4.4 & 0 \\
\hline tai_15_15_2 & 918 & 918 & 918 & 21.1 & 918 & 9.1 & - & 567 & 26.5 & 2190 \\
\hline tai_15_15_3 & 871 & 871 & 871 & 14.3 & 871 & 4.3 & 611.6 & 543 & 3.4 & 0 \\
\hline tai_15_15_4 & 934 & 934 & 934 & 14.2 & 934 & 3.9 & 570.1 & 560 & 1.7 & 0 \\
\hline tai_15_15_5 & 946 & 946 & 946 & 25.7 & 946 & 5.7 & 556.3 & 541 & 8.5 & 1760 \\
\hline tai_15_15_6 & 933 & 933 & 933 & 16.6 & 933 & 4.7 & 574.5 & 560 & 3.0 & 0 \\
\hline tai_15_15_7 & 891 & 891 & 891 & 20.1 & 891 & 10.4 & 724.6 & 566 & 16.5 & 1896 \\
\hline tai_15_15_8 & 893 & 893 & 893 & 14.2 & 893 & 17.3 & 614.0 & 546 & 1.3 & 0 \\
\hline tai_15_15_9 & 899 & 899 & 899.7 & 4.1 & 899.2 & 26.6 & 646.9 & 568 & 39.2 & 4053 \\
\hline tai_15_15_10 & 902 & 902 & 902 & 18.1 & 902 & 6.9 & 720.1 & 586 & 22.9 & 2081 \\
\hline tai_20_20_1 & 1155 & 1155 & 1155 & 54.1 & 1155 & 16.6 & 3519.8 & 3105 & 32.4 & 3340 \\
\hline tai_20_20_2 & 1241 & 1241 & 1241 & 79.7 & 1241 & 23.5 & - & 3559 & 588.4 & 45606 \\
\hline tai_20_20_3 & 1257 & 1257 & 1257 & 48.6 & 1257 & 19.6 & 4126.3 & 2990 & 3.0 & 0 \\
\hline tai_20_20_4 & 1248 & 1248 & 1248 & 49.1 & 1248 & 19.6 & - & 3442 & 2.7 & 0 \\
\hline tai_20_20_5 & 1256 & 1256 & 1256 & 49.1 & 1256 & 19.6 & 3247.3 & 3603 & 3.7 & 0 \\
\hline tai_20_20_6 & 1204 & 1204 & 1204 & 49.3 & 1204 & 19.6 & 3393.0 & 2741 & 10.2 & 1879 \\
\hline tai_20_20_7 & 1294 & 1294 & 1294 & 65.0 & 1294 & 25.4 & 2954.8 & 2912 & 86.9 & 8620 \\
\hline tai_20_20_8 & 1169 & 1171 & 1170.3 & 27.9 & 1170 & 50.9 & - & 2990 & 305.8 & 25503 \\
\hline tai_20_20_9 & 1289 & 1289 & 1289 & 48.6 & 1289 & 78.2 & 3593.8 & 3204 & 1.7 & 0 \\
\hline tai_20_20_10 & 1241 & 1241 & 1241 & 48.8 & 1241 & 78.2 & 4936.2 & 3208 & 1.1 & 0 \\
\hline
\end{tabular}

Table 3: Results for the Taillard benchmark.

benchmark as it only found eight optimal solutions among instances of size $7 \times 7$ and $10 \times 10$, and did not provide results for larger instances. The solution times of RRCP are roughly similar to metaheuristics's with the exception of tai_20_20_02 and tai_20_20_08. Note however that, none of the metaheuristics were able to consistently find the optimum of tai_20_20_08. Results also show that CROSH was more efficient than complex metaheuristics on many large instances (if the average number of nodes $\bar{n}$ is nil, then all runs of CROSH reached the optimum).

BB-Do was the first exact method to solve all $10 \times 10$ instances and most of the $15 \times 15$ and $20 \times 20$. Its bottom-up algorithm clearly outperformed its top-down algorithm on this benchmark because it needed to solve a unique feasible problem to prove optimality. The tight initial upper bound given by CROSH compensates here the drawback of using a 
top-down algorithm. Furthermore, the diversification provided by the randomization and restart mechanism helps to escape from bad initial choices whereas some instances remained unsolved by BB-Do such as tai_15_15_02. With equivalent computational platforms, RRCP clearly outperforms SAT-Ta, the first exact method to close the benchmark. However, the total CPU time of SAT-Ta linearly fits with the number of clauses on this benchmark, whereas it is not necessarily the case with our algorithm. For example, tai_20_20_08 is not more difficult than others $20 \times 20$ as opposed to other approaches. Last, MCS-Lab did not report results on this benchmark.

\begin{tabular}{|c|c|c|c|c|c|c|c|c|c|c|c|c|}
\hline & & \multicolumn{7}{|c|}{ Metaheuristics } & \multicolumn{4}{|c|}{ Exact Algorithms } \\
\hline \multicolumn{2}{|c|}{ Instance } & GA-Pri & \multicolumn{3}{|c|}{ ACO-Blu } & \multicolumn{3}{|c|}{ PSO-Sha } & \multirow{2}{*}{$\frac{\text { BB-Do }}{t}$} & \multirow{2}{*}{$\frac{\mathrm{SAT}-\mathrm{Ta}}{t}$} & \multicolumn{2}{|c|}{ RRCP } \\
\hline Name & Opt & & Best & Avg & $\bar{t}$ & Best & Avg & $\bar{t}$ & & & $\bar{t}$ & $\bar{n}$ \\
\hline j6-per0-0 & 1056 & 1080 & 1056 & 1056 & 27.4 & 1056 & 1056 & 42.1 & 133.0 & 817 & 38.7 & 11032 \\
\hline j6-per0-1 & 1045 & 045 & 1045 & 1049.7 & 61.3 & 1045 & 1045 & 59.7 & 5.2 & 57 & 0.3 & 198 \\
\hline j6-per0-2 & 1063 & 079 & 1063 & 1063 & 38.8 & 1063 & 1063 & 72.6 & 18.0 & 57 & 0.6 & 223 \\
\hline j6-per10-0 & 1005 & 016 & 1005 & 1005 & 10.6 & 1005 & 1005 & 45 & 14.4 & 52 & 0.8 & 263 \\
\hline j6-per10-1 & 1021 & 1036 & 1021 & 1021 & 11.3 & 1021 & 1021 & 21.0 & 4.6 & 46 & 0.3 & 177 \\
\hline j6-per $10-2$ & 1012 & 1012 & 1012 & 1012 & 1.4 & 1012 & 1012 & 8.5 & 13.8 & 51 & 0.5 & 188 \\
\hline j6-per $20-0$ & 1000 & 1018 & 1000 & 1003.6 & 31.1 & 1000 & 1000 & 77.5 & 10.7 & 60 & 0.4 & 208 \\
\hline j6-per20-1 & 1000 & 1000 & 1000 & 1000 & 0.8 & 1000 & 1000 & 1.5 & 0.4 & 46 & 0.2 & 161 \\
\hline j6-per20-2 & 1000 & 1001 & 1000 & 1000 & 3.9 & 1000 & 1000 & 30.6 & 1.0 & 40 & 0.4 & 179 \\
\hline j7-per0-0 & 1048 & 1071 & 1048 & 1052.7 & 207.9 & 1050 & 1051.2 & 104.9 & $(1058)$ & $M$ & 7777.2 & 1564192 \\
\hline j7-per0-1 & 1055 & 1076 & 1057 & 1057.8 & 91.6 & $\dagger 1055$ & 1058.8 & 155.8 & 9421.8 & 428 & 16.5 & 3265 \\
\hline j7-per0-2 & 1056 & 1082 & 1058 & 1059 & 175.9 & 1056 & 1057 & 124.5 & 9273.5 & 292 & 16.4 & 3120 \\
\hline j7-per10-0 & 1013 & 1036 & 1013 & 1016.7 & 217.6 & 1013 & 1016.1 & 183.8 & 2781.9 & 332 & 19.1 & 3981 \\
\hline j7-per10-1 & 1000 & 010 & 1000 & 1002.5 & 189.9 & 1000 & 1000 & 81 & 1563.0 & 121 & 6.4 & 1276 \\
\hline j7-per10-2 & 1011 & 1035 & 1016 & 1019.4 & 180.7 & 1013 & 1014.9 & 125.6 & 15625.1 & 1786 & 583.1 & 128289 \\
\hline j7-per20-0 & 1000 & 1000 & 1000 & 1000 & 0.4 & 1000 & 1000 & 1.9 & 48.8 & 66 & 0.1 & 0 \\
\hline j7-per20-1 & 1005 & 1030 & 1005 & 1007.6 & 259.1 & 1007 & 1008 & 143.2 & 318.8 & 132 & 8.9 & 2130 \\
\hline j7-per $20-2$ & 1003 & 1020 & 1003 & 1007.3 & 257.3 & 1003 & 1004.7 & 160.9 & 2184.9 & 132 & 13.8 & 3150 \\
\hline j8-per0-1 & 1039 & 1075 & 1039 & 1048.7 & 313.5 & 1039 & 1043.3 & 220.8 & & $M$ & 11168.9 & $\overline{1648700}$ \\
\hline j8-per0-2 & 1052 & 1073 & 1052 & 1057.1 & 323.4 & 1052 & 1053.6 & 271.9 & & 870 & 61.3 & 9379 \\
\hline j8-per10-0 & 1017 & 1053 & 1020 & 1026.9 & 346.5 & 1020 & 1026.1 & 205.0 & & 2107 & 184.5 & 24548 \\
\hline j8-per10-1 & 1000 & 1029 & 1004 & 1012.4 & 308.9 & 1002 & 1007.6 & 202.2 & & 8346 & 1099.3 & 165875 \\
\hline j8-per10-2 & 1002 & 1027 & 1009 & 1013.7 & 399.4 & 1002 & 1006 & 162.8 & & 7789 & 4596.5 & 673451 \\
\hline j8-per20-0 & 1000 & 1015 & 1000 & 1001 & 237.2 & 1000 & 1000.6 & 136.9 & & 148 & 9.1 & 2104 \\
\hline j8-per20-1 & 1000 & 1000 & 1000 & 1000 & 2.6 & 1000 & 1000 & 4.5 & & 136 & 0.4 & 128 \\
\hline j8-per20-2 & 1000 & 1014 & 1000 & 1000.6 & 286.2 & 1000 & 1000 & 105.8 & & 144 & 6.7 & 1512 \\
\hline
\end{tabular}

Table 4: Results for the Brucker benchmark.

Results for the Brucker Instances (Table 4) As a result of the relatively low difficulty of the Taillard instances, the Brucker instances were generated. GA-Pri is the most degraded method and reached optimality only five times. ACO-Blu and PSO-Sha showed higher average solving times than on Taillard instances, and some optimal solutions are never reached. On the contrary, CNR-Cha reported improving 3 best-known solutions, and finding 17 optimal solutions. RRCP exhibits good performance against metaheuristics with the exception of several instances where the comparison is hard to achieve because solving times are greater but metaheuristics fail to return optimal solutions at each run.

The top-down algorithm of BB-Do solved eight $7 \times 7$ instances of the nine. MCS-Lab closed three of the six remaining instances (j8-per0-2, j8-per10-0, and j8-per10-1) and SAT-Ta closed later the three last instances (j7-per0-0, j8-per0-1, and j8-per10-2). 
With the exception of j7-per10-2, j8-per10-0 and j8-per10-1, RRCP yields solving times below or similar to estimated runtimes of MCS-Lab which range from 5 to 35 seconds. Solving times of SAT-Ta stay greater than RRCP's, especially for j7-per0-0 and j8-per0-1 where their experiments required great computation time. On this benchmark, their solving times do not exhibit a linear behaviour on the problem's size.

\begin{tabular}{|c|c|c|c|c|c|c|c|c|c|c|c|c|}
\hline & & \multicolumn{7}{|c|}{ Metaheuristics } & \multicolumn{4}{|c|}{ Exact Algorithms } \\
\hline \multicolumn{2}{|c|}{ Instance } & \multirow[t]{2}{*}{ GA-Pri } & \multicolumn{3}{|c|}{ ACO-Blu } & \multicolumn{3}{|c|}{ PSO-Sha } & \multicolumn{2}{|c|}{ BB-Gue SAT-Ta } & \multicolumn{2}{|c|}{ RRCP } \\
\hline Name & Opt & & Best & Avg & $\bar{t}$ & Best & Avg & $\bar{t}$ & & $t$ & $\bar{t}$ & $\bar{n}$ \\
\hline gp06-01 & 1264 & 264 & 1264 & 1264.7 & 30.8 & 1264 & 1264 & 176.1 & 264 & 57 & 0.3 & 80 \\
\hline & 1285 & 285 & 1285 & 1285.7 & 48.7 & 1285 & 1285 & 147.8 & 285 & 65 & 0.2 & 172 \\
\hline gp06-03 & 1255 & 255 & 1255 & 1255 & 30.0 & 1255 & 1255.6 & 133.1 & 255 & 72 & 0.1 & 124 \\
\hline gp06-04 & 1275 & 1275 & 1275 & 1275 & 25.9 & 1275 & 1275 & 60.8 & 1275 & & 0.1 & 67 \\
\hline gp06-05 & 1299 & 1300 & 1299 & 1299.2 & 39.9 & 1299 & 1299 & 159.6 & 1299 & 6 & 0.1 & 67 \\
\hline gp06-06 & 1284 & 1284 & 1284 & 1284 & 43 & 1284 & 1284 & 109.4 & 1284 & 5 & 0.1 & 68 \\
\hline gp06-07 & 1290 & 1290 & 1290 & 1290 & 10.5 & 1290 & 1290 & 1.6 & 1290 & 77 & 0.1 & 63 \\
\hline gp06-08 & 1265 & 1266 & 1265 & 1265.2 & 71.9 & 1265 & 1265.5 & 134.3 & 1265 & 71 & 0.1 & 52 \\
\hline gp06-09 & 1243 & 1243 & 1243 & 1243 & 9.8 & 1243 & 1243.1 & 156.5 & 1264 & 72 & 0.2 & 170 \\
\hline gp06-10 & 1254 & 1254 & 1254 & 1254 & 4.6 & 1254 & 1254 & 79.8 & 1254 & 57 & 0.3 & 241 \\
\hline gp07-01 & 1159 & 1159 & 1159 & 1159 & 86.9 & 1159 & 1159.3 & 223.7 & 1160 & 99 & 0.9 & 367 \\
\hline gp07-02 & 1185 & 185 & 1185 & 1185 & 80.3 & & 1185 & 1.2 & 1191 & 148 & 0.6 & \\
\hline $07-03$ & 1237 & 237 & 1237 & 1237 & 40.9 & 37 & 1237 & 9.5 & 1242 & 132 & 0.7 & 54 \\
\hline $07-04$ & 1167 & 167 & 1167 & 1167 & 59.2 & 1167 & 1167 & 160.4 & 1167 & 13 & 0.7 & 144 \\
\hline $7-05$ & 1157 & 157 & 1157 & 1157 & 124.4 & 1157 & 1157 & 139 & 1191 & & 0.8 & \\
\hline $7-06$ & 1193 & 193 & 1193 & 1193.9 & 152.4 & 1193 & 1193.1 & 198 & 200 & & .8 & 306 \\
\hline 07 & 1185 & 85 & & 1185. & & & & 1 & 1201 & & 0.6 & 48 \\
\hline $07-08$ & 1180 & 181 & 1180 & 1181.4 & 206.7 & 1180 & 1180 & 139.4 & 1183 & 144 & 0.7 & 117 \\
\hline $07-09$ & 1220 & 1220 & 1220 & 1220.1 & 127.9 & 1220 & 1220 & 143.9 & 1220 & 150 & 0.7 & 177 \\
\hline gp07-10 & 1270 & 1270 & 1270 & 1270.1 & 65.6 & 1270 & 1270 & 0.5 & 1270 & 127 & 0.6 & \\
\hline gp08-01 & 1130 & 1160 & 1130 & 1132.4 & 335.0 & 1130 & 1140.3 & 277.3 & 1195 & 160 & 2.6 & 1485 \\
\hline $08-02$ & 1135 & 136 & 1135 & 1136.1 & 228.4 & 1135 & 1135.4 & 258.3 & 1197 & 190 & 1.2 & 304 \\
\hline $08-03$ & 1110 & 111 & 111 & 1113.7 & 336.3 & 1110 & 1114 & 240.3 & 1158 & 197 & 1.6 & 622 \\
\hline gp08-04 & 1153 & 1168 & 154 & 1156 & 275.7 & 1153 & 1153.2 & 308.1 & 1168 & 227 & 1.4 & 566 \\
\hline gp08-05 & 1218 & 1218 & 1219 & 1219.8 & 347.7 & 1218 & 1218.9 & 56.6 & 1218 & 247 & 1.2 & 206 \\
\hline gp08-06 & 1115 & 28 & 1116 & 1123.2 & 359.2 & 1115 & 1126.9 & 249.6 & 1171 & 175 & 2.3 & 1498 \\
\hline gp08-07 & 1126 & 128 & 1126 & 1134.6 & 296.8 & 1126 & 1129.8 & 287.3 & 1157 & 204 & 3.6 & 2775 \\
\hline gp08-08 & 1148 & 148 & 148 & 1149 & 277.4 & 148 & 1148 & 179.3 & 1191 & 183 & 2.0 & 1281 \\
\hline gp08-09 & 1114 & 120 & 1117 & 1119 & 279.0 & 114 & 1114.3 & 223.6 & 1142 & 189 & 2.0 & 1140 \\
\hline gp08-10 & 1161 & 161 & 1161 & 1161.5 & 281.3 & 161 & 1161.4 & 217.1 & 1161 & 203 & 1.1 & 245 \\
\hline gp09-01 & 1129 & 1143 & 1135 & 1142.8 & 412.9 & 129 & 1133.2 & 376.3 & 1150 & 323 & 3.6 & 1691 \\
\hline $09-02$ & 1110 & 14 & 12 & 1113.7 & 430.8 & $\dagger 1110$ & 1114.1 & 335.9 & 1226 & 327 & 10.7 & 8000 \\
\hline $09-03$ & 1115 & 18 & 18 & 1120.4 & 428.0 & & 117 & 31 & 150 & & 2.8 & 1422 \\
\hline gp09-04 & 1130 & & & 1140 & & & 1135.8 & 328 & 1181 & 340 & 4.3 & 2219 \\
\hline & 1180 & 180 & & 1180.5 & 29 & & 1180 & 22.3 & 1180 & 36 & 1.7 & 266 \\
\hline-06 & 1093 & & & 1195.6 & 38 & & 10 & 277 & 136 & 4 & 4.6 & 2387 \\
\hline & 1090 & & & 1101.4 & 43 & & & & 173 & & 5.9 & 3483 \\
\hline & 1105 & & & 1113.7 & 376 & 108 & 1108.3 & & 1193 & & 3.1 & 1446 \\
\hline $9-09$ & 1123 & 32 & 127 & 1132.5 & 402.6 & $\dagger 1123$ & 1126.5 & 358.6 & 1218 & 316 & 3.2 & 1537 \\
\hline $9-10$ & 1110 & 130 & 1120 & 1126.3 & 435.8 & $\dagger 1112$ & 1126.5 & 297.7 & 1166 & 355 & 6.1 & 2784 \\
\hline $0-01$ & 1093 & & 099 & 1109 & 567.5 & 1093 & 1096.8 & 455.7 & 1151 & 470 & 29.8 & 6661 \\
\hline $0-02$ & 1097 & & 101 & 1107.4 & 501.7 & 097 & 1099.1 & 382.7 & 1178 & 526 & 9.7 & 3140 \\
\hline $0-03$ & 1081 & & 1082 & 1098 & 658.7 & $\dagger 1081$ & 1090.3 & 450.8 & 1162 & 535 & 13.6 & 4196 \\
\hline $0-04$ & 1077 & 90 & 1093 & 1096.6 & 588.1 & 1083 & 1092.1 & 371.8 & 1165 & 515 & 12.4 & 3921 \\
\hline $0-05$ & 1071 & 994 & 1083 & 1092.4 & 636.4 & $\dagger 1073$ & 1092.2 & 314.1 & 1125 & 515 & 16.3 & 4782 \\
\hline $0-06$ & 1071 & 074 & 1088 & 1104.6 & 595.5 & 1071 & 1074.3 & 289.7 & 1179 & 508 & 12.4 & 3894 \\
\hline $0-07$ & 1079 & 0 & 1084 & 1091.5 & 389.6 & $\dagger 1080$ & 1081.1 & 167.4 & 1172 & 523 & 8.7 & 2188 \\
\hline $0-08$ & 1093 & 098 & 1099 & 1104.8 & 615.9 & $\dagger 1095$ & 1097.6 & 324.5 & 1181 & 498 & 10.5 & 3477 \\
\hline $10-09$ & 1112 & 1121 & 1121 & 1128.7 & 554.5 & $\dagger 1115$ & 1127 & 428.2 & 1188 & 541 & 10.1 & 3303 \\
\hline gp10-10 & 1092 & 1095 & 1097 & 1106.7 & 562.5 & 1092 & 1094 & 487.9 & 1172 & 656 & 7.4 & 1724 \\
\hline
\end{tabular}

Table 5: Results for the Guéret-Prins benchmark. 
Results for the Guéret-Prins Instances (Table 5) The Guéret-Prins instances seems to be more difficult to solve because the metaheuristics are less effective. Average results of ACO-Blu and PSO-Sha decrease according to problem size. In spite of higher solving times, fewer optimums are reached, especially with ACO-Blu. By comparison, GA-Pri is more effective than with Brucker benchmark and CNR-Cha claims to improve 12 solutions. RRCP is particularly well-suited for this series of problems and it prevails over all metaheuristics in every problem size.

BB-Gue solved most instances to optimality up to size $6 \times 6$ as well as a few large instances. BB-Do did not report results on this benchmark and MCS-Lab reported closing all the instances, but again did not report detailed solution times. Solving times of RRCP are always lower than estimated runtimes of MCS-Lab which range from 40 to 50 seconds. Solving times of SAT-Ta decrease and again linearly fit the number of clauses, but do not compete with RRCP's.

\section{Conclusion}

We have presented a constraint programming algorithm to solve the Open-Shop problem. This algorithm consists of computing an initial upper bound before solving a high-level declarative model (tasks, resources, precedences) by a top-down branch-and-bound enhanced with randomization and restart.

The computational results matched the metaheuristics for the Taillard benchmark, whereas we obtained better solution quality with solution times that are orders of magnitude lower than the metaheuristics for the Brucker and Guéret-Prins benchmarks. The computational results also outperformed all exact approaches that reported detailed solution times.

In further research, we will apply other branching schemes. In addition, subsequent research topics include the study of other shop problems such as Flow-Shop Problems and Job-Shop Problems.

\section{References}

Baptiste, Philippe, Claude Le Pape. 1996. Edge-finding constraint propagation algorithms for disjunctive and cumulative scheduling. Proceedings of the Fifteenth Workshop of the U.K. Planning Special Interest Group.

Beck, J. Christopher, Andrew J. Davenport, Edward M. Sitarski, Mark S. Fox. 1997. Texturebased heuristics for scheduling revisited. AAAI/IAAI. 241-248.

Blum, Christian. 2005. Beam-ACO: hybridizing ant colony optimization with beam search: an application to open shop scheduling. Comput. Oper. Res. 32 1565-1591.

Bräsel, H., T. Tautenhahn, F. Werner. 1993. Constructive heuristic algorithms for the open shop problem. Computing 51 95-110.

Brucker, P., T. Hilbig, J. Hurink. 1996. A branch and bound algorithm for scheduling problems with positive and negative time-lags. Tech. rep., Osnabrueck University. 
Brucker, Peter, Andreas Drexl, Rolf Mohring, Klaus Neumann, Erwin Pesch. 1999. Resourceconstrained project scheduling: Notation, classification, models, and methods. European Journal of Operational Research 112 3-41.

Brucker, Peter, Johann Hurink, Bernd Jurisch, Birgit Wöstmann. 1997. A branch \& bound algorithm for the open-shop problem. GO-II Meeting: Proceedings of the second international colloquium on Graphs and optimization. Elsevier Science Publishers B. V., Amsterdam, The Netherlands, 43-59.

Carlier, J., E. Pinson. 1994. Adjustment of heads and tails for the job-shop problem. European Journal of Operational Research 78 146-161.

Caseau, Y., F. Laburthe. 1995. Disjunctive scheduling with task intervals. Tech. rep., Laboratoire d'Informatique de I'Ecole Normale Superieure.

Cesta, A., A. Oddi. 1996. Gaining efficiency and flexibility in the simple temporal problem. TIME '96: Proceedings of the 3rd Workshop on Temporal Representation and Reasoning (TIME'96). IEEE Computer Society, Washington, DC, USA, 45.

Chatzikokolakis, Konstantinos, George Boukeas, Panagiotis Stamatopoulos. 2004. Construction and repair: A hybrid approach to search in csps. SETN 30252004.

Choco team. 2008. Choco: an open source java constraint programming library. URL http://choco.emn.fr.

Dechter, Rina. 2003. Temporal constraint networks. Constraint Processing. Morgan Kaufmann, San Francisco, 333-362.

Dorndorf, U., E. Pesch, T. Phan Huy. 2001. Solving the open shop scheduling problem. Journal of Scheduling 4 157-174.

Frigioni, Daniele, Tobias Miller, Umberto Nanni, Christos D. Zaroliagis. 2001. An experimental study of dynamic algorithms for transitive closure. ACM Journal of Experimental Algorithms 69.

Gondran, M., M. Minoux. 1984. Graphs and Algorithms. John Wiley \& Sons, New York.

Gonzalez, T., S. Sahni. 1976. Open shop scheduling to minimize finish time. Journal of the Association for Computing Machinery 23 665-679.

Guéret, C., C. Prins. 1999. A new lower bound for the open shop problem. Annals of Operations Research 92 165-183.

Guéret, Christelle. 1997. Problèmes d'ordonnancement sans contraintes de précédence. Thèse, Université de Technologie de Compiègne. Codirecteurs : C. Prins et J. Carlier.

Guéret, Christelle, Narendra Jussien, Christian Prins. 2000. Using intelligent backtracking to improve branch-and-bound methods: An application to open-shop problems. European Journal of Operational Research 127 344-354. 
Guéret, Christelle, Christian Prins. 1998. Forbidden intervals for open-shop problems. Tech. rep., École des Mines de Nantes.

Kellerer, H., U. Pferschy, D. Pisinger. 2004. Knapsack Problems. Springer, Berlin, Germany.

Kolisch, Rainer. 1996. Serial and parallel resource-constrained project scheduling methods revisited: Theory and computation. European Journal of Operational Research 90320 333.

Laborie, Philippe. 2005. Complete mcs-based search: Application to resource constrained project scheduling. Leslie Pack Kaelbling, Alessandro Saffiotti, eds., IJCAI. Professional Book Center, 181-186.

Le Pape, Claude, Philippe Couronne, Didier Vergamini, Vincent Gosselin. 1994. Timeversus-capacity compromises in project scheduling. Proceedings of the Thirteenth Workshop of the U.K. Planning Special Interest Group.

Lecoutre, Christophe, Lakhdar Sais, Sébastien Tabary, Vincent Vidal. 2007. Nogood recording from restarts. Manuela M. Veloso, ed., IJCAI 2007, Proceedings of the 20th International Joint Conference on Artificial Intelligence, Hyderabad, India, January 6-12, 2007. $131-136$.

Luby, Sinclair, Zuckerman. 1993. Optimal speedup of las vegas algorithms. IPL: Information Processing Letters 47 173-180.

Pearce, David J., Paul H. J. Kelly. 2006. A dynamic topological sort algorithm for directed acyclic graphs. ACM Journal of Experimental Algorithms 11 1-7.

Prins, Christian. 2000. Competitive genetic algorithms for the open-shop scheduling problem. Mathematical methods of operations research 52 389-411.

Sha, D. Y., Cheng-Yu Hsu. 2008. A new particle swarm optimization for the open shop scheduling problem. Comput. Oper. Res. 35 3243-3261.

Taillard, E. 1993. Benchmarks for basic scheduling problems. European Journal of Operations Research $64278-285$.

Tamura, Naoyuki, Akiko Taga, Satoshi Kitagawa, Mutsunori Banbara. 2006. Compiling finite linear CSP into SAT. Principles and Practice of Constraint Programming - CP 2006, Lecture Notes in Computer Science, vol. 4204. Springer Berlin / Heidelberg, 590603.

Vilím, Petr. 2004. O(n log n) filtering algorithms for unary resource constraint. CPAIOR 2004, Nice, France, April 20-22, 2004, Lecture Notes in Computer Science, vol. 3011. Springer, 335-347.

Walsh, Toby. 1999. Search in a small world. IJCAI '99: Proceedings of the Sixteenth International Joint Conference on Artificial Intelligence. Morgan Kaufmann Publishers Inc., San Francisco, CA, USA, 1172-1177. 
Wu, Huayue, Peter van Beek. 2007. On universal restart strategies for backtracking search. Principles and Practice of Constraint Programming CP 200\%, Lecture Notes in Computer Science, vol. 4741/2007. Springer Berlin / Heidelberg, 681-695. 